\title{
A Hybrid Method for Planning and Scheduling
}

\author{
J. N. Hooker \\ Carnegie Mellon University \\ jh38@andrew.cmu.edu
}

April 2004

\begin{abstract}
We combine mixed integer linear programming (MILP) and constraint programming (CP) to solve planning and scheduling problems. Tasks are allocated to facilities using MILP and scheduled using CP, and the two are linked via logic-based Benders decomposition. Tasks assigned to a facility may run in parallel subject to resource constraints (cumulative scheduling). We solve minimum cost problems, as well as minimum makespan problems in which all tasks have the same release date and deadline. We obtain computational speedups of several orders of magnitude relative to the state of the art in both MILP and CP.
\end{abstract}

We address a fundamental class of planning and scheduling problems for manufacturing and supply chain management. Tasks must be assigned to facilities and scheduled subject to release dates and deadlines. Tasks may run in parallel on a given facility provided the total resource consumption at any time remains with limits (cumulative scheduling). In our study the objective is to minimize cost or minimize makespan.

The problem naturally decomposes into an assignment portion and a scheduling portion. We exploit the relative strengths of mixed integer linear programming (MILP) and constraint programming (CP) by applying MILP to the assignment problem and CP to the scheduling problem. We then link the two with a logic-based Benders algorithm.

We obtain speedups of several orders of magnitude relative to the existing state of the art in both mixed integer programming (CPLEX) and constraint programming (ILOG Scheduler). As a result we solve larger instances to optimality than could be solved previously.

\section{The Basic Idea}

Benders decomposition solves a problem by enumerating values of certain primary variables. For each set of values enumerated, it solves the subproblem that results from fixing the primary variables to these values. Solution of the subproblem generates a Benders cut (a type of nogood) that the primary variables must satisfy in all subsequent solutions enumerated. The next set of values for the primary variables is obtained by solving the master problem, which contains all the Benders cuts so far generated. 
In this paper, the primary variables define the assignment of tasks to facilities, and the master problem is the assignment problem augmented with Benders cuts. The subproblem is the set of cumulative scheduling problems (one for each facility) that result from a given assignment.

In classical Benders decomposition [1,3], the subproblem is always a continuous linear or nonlinear programming problem, and there is a standard way to obtain Benders cuts. In a logic-based Benders method, the subproblem is an arbitrary optimization problem, and a specific scheme for generating cuts must be devised for each problem class by solving the inference dual of the subproblem. In the present context, the Benders cuts must also be linear inequalities, since the master problem is an MILP. It is also important in practice to augment the master problem with a linear relaxation of the subproblem.

The main contribution of this paper is to develop effective linear Benders cuts and subproblem relaxations for (a) minimum cost problems with cumulative scheduling, and (b) minimum makespan problems with cumulative scheduling in which all tasks have the same release date and deadine.

\section{Previous Work}

Logic-based Benders decomposition was introduced by Hooker and Yan [6] in the context of logic circuit verification. The idea was formally devloped in [4] and applied to 0-1 programming by Hooker and Ottosson [5].

Jain and Grossmann [7] successfully applied logic-based Benders to minimumcost planning and scheduling problems in which the subproblems are one-machine disjunctive (rather than cumulative) scheduling problems. The Benders cuts are particularly simple in this case because the subproblem is a feasibility problem rather than an optimization problem. Two goals of the present paper are (a) to accommodate cumulative scheduling, and (b) to develop Benders cuts when the subproblem is an optimization problem, as in the case of minimum makespan problems.

In related work, we observed in [4] that the master problem need only be solved once by a branching algorithm that accumulates Benders cuts as they are generated. Thorsteinsson [8] showed that this approach, which he called branch and check, can result in substantially better performance on the Jain and Grossmann problems than standard logic-based Benders. We did not implement branch and check for this study because it would require hand coding of a branchand-cut algorithm for the master problem. But we obtained substantial speedups without it.

Classical Benders decomposition can also be useful in a CP context, as shown by Eremin and Wallace [2].

\section{The Problem}

The planning and scheduling problem may be defined as follows. Each task $j \in\{1, \ldots, n\}$ is to be assigned to a facility $i \in\{1, \ldots m\}$, where it consumes 
processing time $p_{i j}$ and resources at the rate $c_{i j}$. Each task $j$ has release time zero and deadline $d_{j}$ (viewed as a due date in the case of minimum tardiness problems). The tasks assigned to facility $i$ must be given start times $t_{j}$ in such a way that the total rate of resource consumption on facility $i$ is never more than $C_{i}$ at any given time.

We investigate two objective functions. If we let $x_{j}$ be the facility assigned to task $j$, the cost objective is $g(x, t)=\sum_{j} f_{x_{j} j}$, where $f_{i j}$ is the fixed cost of processing task $j$ on facility $i$. The makespan objective is $g(x, t)=\min _{j}\left\{t_{j}+\right.$ $\left.p_{x_{j} j}\right\}$.

\section{Constraint Programming Formulation}

The problem is succintly written using the constraint cumulative $(t, p, c, C)$, which requires that tasks be scheduled at times $t=\left(t_{1}, \ldots, t_{n}\right)$ so that the total rate of resource consumption at any given time never exceeds $C$. Thus $\sum_{j \in J_{t}} c_{j} \leq C$ for all $t$, where $J_{t}=\left\{j \mid t_{j} \leq t \leq t_{j}+p_{j}\right\}$ is the set of tasks underway at time $t$.

The planning and scheduling problem becomes

$$
\begin{array}{ll}
\operatorname{minimize} & g(x, t) \\
\text { subject to } & \text { cumulative }\left(\left(t_{j} \mid x_{j}=i\right),\left(p_{i j} \mid x_{j}=i\right),\left(c_{i j} \mid x_{j}=i\right), C_{i}\right) \text {, all } i \\
& r_{j} \leq t_{j} \leq d_{j}-p_{x_{j} j}, \text { all } j
\end{array}
$$

where $g(x, t)$ is the desired objective function and $\left(t_{j} \mid x_{j}=i\right)$ denotes the tuple of start times for tasks assigned to facility $i$. The second constraint enforces the time windows.

\section{Mixed Integer Programming Formulation}

The most straightforward MILP formulation discretizes time and enforces the resource capacity constraint at each discrete time. Let the $0-1$ variable $x_{i j t}=1$ if task $j$ starts at discrete time $t$ on facility $i$. The formulation is

$$
\begin{array}{ll}
\min & g(x, t) \\
\text { subject to } & \sum_{i t} x_{i j t}=1, \text { all } j \\
& \sum_{j} \sum_{t^{\prime} \in T_{i j t}} c_{i j} x_{i j t} \leq C_{i}, \text { all } i, t \\
& x_{i j t}=0, \text { all } j, t \text { with } d_{j}-p_{i j}<t \leq r_{j} \text { or } t>n-p_{i j}+1(c)
\end{array}
$$

where $T_{i j t}=\left\{t^{\prime} \mid t-p_{i j}<t^{\prime} \leq t\right\}$ is the set of discrete times at which a task $j$ in progress on facility $i$ at time $t$ might start processing. Constraint (a) ensures that each task starts once on one facility, (b) enforces the resource limit, and (c) the time windows. The cost objective is $g(x, t)=\sum_{i j t} f_{i j} x_{i j t}$. The makespan objective is $g(x, t)=z$, together with the constraints $z \geq \sum_{i t}\left(t+p_{i j}\right) x_{i j t}$ for all $j$. 
Due to the size of (2), we also investigated a smaller discrete event model suggested by [9], which uses continuous time. However, it proved to be much harder to solve than (2). We therefore omitted the discrete event model from the computational studies described below.

\section{Logic-based Benders Decomposition}

Logic-based Benders decomposition applies to problems of the form

$$
\begin{aligned}
\operatorname{minimize} & f(x, t) \\
\text { subject to } & C(x, t) \\
& x \in D_{x}, t \in D_{t}
\end{aligned}
$$

where $C(x, t)$ is a set of constraints containing variables $x, t . D_{x}$ and $D_{y}$ denote the domains of $x$ and $y$, respectively. When $x$ is fixed to a given value $\bar{x} \in D_{x}$, the following subproblem results:

$$
\begin{aligned}
\operatorname{minimize} & f(\bar{x}, t) \\
\text { subject to } & C(\bar{x}, t) \\
& t \in D_{t}
\end{aligned}
$$

Here $C(\bar{x}, t)$ is the constraint that results from fixing $x=\bar{x}$ in $C(x, t)$.

The inference dual of (4) is the problem of inferring the tightest possible lower bound on $f(\bar{x}, t)$ from $C(\bar{x}, t)$. It can be written

$$
\begin{gathered}
\text { maximize } \\
\text { subject to } \\
\qquad(\bar{x}, t) \Longrightarrow f(\bar{x}, t) \geq v \\
v \in \mathbb{R}
\end{gathered}
$$

where $\Longrightarrow$ means "implies" (see [4] for details).

The solution of the dual can be viewed as a derivation of the tightest possible bound $\hat{v}$ on $f(x, t)$ when $x=\bar{x}$. For purposes of Benders decomposition, we wish to derive not only a bound when $x=\bar{x}$ but a function $B_{\bar{x}}(x)$ that provides a valid lower bound on $f(x, t)$ for any given $x \in D_{x}$. In particular, $B_{\bar{x}}(\bar{x})=\hat{v}$. If $z$ is the objective function value of (3), this bounding function provides the valid inequality $z \geq B_{\bar{x}}(x)$, which we call a Benders cut.

In iteration $H$ of the Benders algorithm, we solve a master problem whose constraints are the Benders cuts so far generated:

$$
\begin{array}{ll}
\min & z \\
\text { subject to } & z \geq B_{x^{h}}(x), h=1, \ldots, H-1 \\
& z \in \mathbb{R}, x \in D_{x}
\end{array}
$$

Here $x^{1}, \ldots, x^{H-1}$ are the solutions of the previous $H-1$ master problems. Then the solution $\bar{x}$ of (6) defines the next subproblem (4). 
If we let $v_{1}^{*}, \ldots, v_{H-1}^{*}$ denote the optimal values of the previous $H-1$ subproblems, the algorithm continues until the optimal value $z_{H}^{*}$ of the master problem equals $v^{*}=\min \left\{v_{1}^{*}, \ldots, v_{H-1}^{*}\right\}$. It is shown in $[4,5]$ that the algorithm converges finitely to an optimal solution under fairly weak conditions, which hold in the present case. At any point in the algorithm, $z_{H}^{*}$ and $v^{*}$ provide lower and upper bounds on the optimal value of the problem.

In the planning and scheduling problem (1), any assignment $\bar{x}$ of tasks to facilities creates the subproblem:

$$
\begin{array}{ll}
\min & g(\bar{x}, t) \\
\text { subject to } & \text { cumulative }\left(\left(t_{j} \mid \bar{x}_{j}=i\right),\left(p_{i j} \mid \bar{x}_{j}=i\right),\left(c_{i j} \mid \bar{x}_{j}=i\right), C_{i}\right), \text { all } i \\
& r_{j} \leq t_{j} \leq d_{j}-p_{\bar{x}_{j} j}, \text { all } j
\end{array}
$$

which decomposes into a separate scheduling problem for each facility. After solving the subproblem we generate a Benders cut that becomes part of the master problem (6).

The bounding function $B_{\bar{x}}(x)$ is generally obtained by examining the type of reasoning that led to a bound for $x=\bar{x}$ and extending this reasoning to obtain a bound for general $x$. In the present context, however, only the primal solution (the schedule itself) is available from the commercial CP solver. We therefore design Benders cuts that require only this information.

\section{Minimizing Cost}

The cost objective presents the simplest case, since cost can be computed in terms of master problem variables, and the subproblem is a feasibility problem. Let $J_{i}^{h}=\left\{j \mid x_{j}^{h}=i\right\}$ be the set of tasks assigned to facility $i$ in iteration $h$. If there is no feasible schedule for facility $i$, the most obvious Benders cut simply rules out assigning this same set $J_{i}^{h}$ of tasks to facility $i$. In this case $B_{x^{h}}(x)$ takes the value $\infty$ when there is an infeasibility and the value $\sum_{j} f_{x_{j} j}$ otherwise. The master problem (5), written as a 0-1 programming problem, becomes

$$
\begin{aligned}
\operatorname{minimize} & \sum_{i j} f_{i j} x_{i j} \\
\text { subject to } & \sum_{i} x_{i j}=1 \text {, all } j \\
& \sum_{j \in J_{i}^{k}}\left(1-x_{i j}\right) \geq 1 \text {, all } i, h=1, \ldots, H-1(b) \\
& \text { relaxation of subproblem }
\end{aligned}
$$

where $x_{i j} \in\{0,1\}$, and where constraints (b) are the Benders cuts.

Experience shows that it is important to include a relaxation of the subproblem within the master problem. A straightforward relaxation can be obtained as follows. For any two times $t_{1}, t_{2}$, let $J\left(t_{1}, t_{2}\right)$ be the set of tasks $j$ whose time windows lie between $t_{1}$ and $t_{2}$; that is, $t_{1} \leq r_{j}$ and $d_{j} \leq t_{2}$. If the tasks 
Let $\mathcal{R}_{i}=\emptyset$.

For $j=1, \ldots, p$ :

Set $k^{\prime}=0$.

For $k=1, \ldots, q$ :

If $r_{k} \geq r_{j}$ and $T^{i}\left(\bar{r}_{j}, \bar{d}_{k}\right)<T^{i}\left(\bar{r}_{j}, \bar{d}_{k^{\prime}}\right)$ then

Remove from $\mathcal{R}_{i}$ all $R^{i}\left(\bar{r}_{j^{\prime}}, \bar{d}_{k}\right)$ for which $T^{i}\left(\bar{r}_{j}, \bar{d}_{k}\right) \geq T^{i}\left(\bar{r}_{j^{\prime}}, \bar{d}_{k}\right)$.

Add $R^{i}\left(\bar{r}_{j}, \bar{d}_{k}\right)$ to $\mathcal{R}_{i}$ and set $k^{\prime}=k$.

Fig. 1. $O\left(n^{3}\right)$ algorithm for generating an inequality set $\mathcal{R}_{i}$ that relaxes the time window constraints for facility $i$. By convention $\bar{d}_{0}=-\infty$.

$j \in J \subset J\left(t_{1}, t_{2}\right)$ are assigned to the same facility $i$, then clearly the "energy consumption" $\sum_{j \in J} p_{i j} c_{i j}$ of these tasks can be at most $C_{i}\left(t_{2}-t_{1}\right)$ if they are to be scheduled in the time interval $\left[t_{1}, t_{2}\right]$. This yields the valid inequality

$$
\frac{1}{C_{i}} \sum_{j \in J\left(t_{1}, t_{2}\right)} p_{i j} c_{i j} x_{i j} \leq t_{2}-t_{1}
$$

which we refer to as inequality $R^{i}\left(t_{1}, t_{2}\right)$. If we let $\bar{r}_{1}, \ldots, \bar{r}_{n_{r}}$ be the distinct elements of $\left\{r_{1}, \ldots, r_{n}\right\}$ in increasing order, and similarly for $\bar{d}_{1}, \ldots, \bar{d}_{n_{d}}$, we have a relaxation consisting of the inequalities

$$
R^{i}\left(\bar{r}_{j}, \bar{d}_{k}\right), \quad j=1, \ldots, n_{r}, k=1, \ldots, n_{d}
$$

for each facility $i$. These inequalities serve as the relaxation (c) in (8).

Many of these inequalities may be redundant of the others, and if desired they can be omitted from the relaxation. Let

$$
T^{i}\left(t_{1}, t_{2}\right)=\frac{1}{C_{i}} \sum_{j \in J\left(t_{1}, t_{2}\right)} p_{i j} c_{i j}-t_{2}+t_{1}
$$

be the tightness of $R^{i}\left(t_{1}, t_{2}\right)$. It is easily verified that $R^{i}\left(t_{1}, t_{2}\right)$ dominates $R^{i}\left(u_{1}, u_{2}\right)$ whenever $\left[t_{1}, t_{2}\right] \subset\left[u_{1}, u_{2}\right]$ and $T^{i}\left(t_{1}, t_{2}\right) \geq T^{i}\left(u_{1}, u_{2}\right)$. A set of undominated inequalities can be generated for each machine using the algorithm of Fig. 1. It has $O\left(n^{3}\right)$ complexity in the worst case, since it is possible that none of the inequalities are eliminated. This occurs, for instance, when each $r_{j}=j-1, d_{j}=j$, and $p_{i j}=2$. However, the algorithm need only be run once as a preprocessing routine.

In practice the relaxation can be simplified by supposing that the release times are all $r_{0}=\min _{j}\left\{r_{j}\right\}$. Then the relaxation (c) in (8) consists of

$$
R^{i}\left(r_{0}, \bar{d}_{k}\right), \quad k=1, \ldots, n_{d}
$$

for each facility $i$. The redundant inequalities can be eliminated running the simple $O(n)$ algorithm of Fig. 2 for each facility. Similarly, one can suppose that the deadlines are all $d_{0}=\max _{j}\left\{d_{j}\right\}$ and use the inequalities $R^{i}\left(\bar{r}_{j}, d_{0}\right)$. 
Let $\mathcal{R}_{i}=\emptyset$, and set $j=0$.

For $k=1, \ldots, p_{d}$ :

If $T^{i}\left(r_{0}, \bar{d}_{k}\right)>T^{i}\left(r_{0}, \bar{d}_{j}\right)$ then add $R^{i}\left(r_{0}, \bar{d}_{k}\right)$ to $\mathcal{R}_{i}$ and set $j=k$.

Fig. 2. $O(n)$ algorithm for generating an inequality set $\mathcal{R}_{i}$ that relaxes the time window constraints for facility $i$, where $r_{0}=\bar{d}_{0}=\min _{j}\left\{r_{j}\right\}$ and $T^{i}\left(r_{0}, r_{0}\right)=0$.

\section{Minimizing Makespan}

This case is less straightforward because the subproblem is an optimization problem. However, when all tasks have the same time window, there are relatively simple linear Benders cuts. We also use a linear subproblem relaxation that is valid for any set of time windows.

The Benders cuts are based on the following fact:

Lemma 1. Consider a minimum tardiness problem $P$ in which tasks $1, \ldots, n$ with release time 0 and deadline $d_{0}$ are to be scheduled on a single facility $i$. Let $M^{*}$ be the minimum makespan for $P$, and $\hat{M}$ the minimum makespan for the problem $\hat{P}$ that is identical to $P$ except that tasks $1, \ldots, s$ are removed. Then $M^{*}-\hat{M} \leq \Delta$ where

$$
\Delta=\sum_{j=1}^{s} p_{i j}
$$

Proof. Consider any optimal solution of $\hat{P}$ and extend it to a solution $S$ of $P$ by scheduling tasks $1, \ldots, s$ sequentially after $\hat{M}$. That is, for $k=1, \ldots, s$ let job $k$ start at time $\hat{M}+\sum_{j=1}^{k-1} p_{i j}$. The makespan of $S$ is $\hat{M}+\Delta$. If $\hat{M}+\Delta \leq d_{0}$, then $S$ is feasible for $P$, so that $M^{*} \leq \hat{M}+\Delta$ and the lemma follows. If $\hat{M}+\Delta>d_{0}$ we know there is a solution of $\bar{P}$ whose makespan $M^{*}<\hat{M}+\Delta$, and again the lemma follows.

The lemma does not hold when the deadlines differ. Consider for example an instance with three tasks where $\left(r_{1}, r_{2}, r_{3}\right)=(0,0,0),\left(d_{1}, d_{2}, d_{3}\right)=(2,1, \infty)$, $\left(p_{i 1}, p_{i 2}, p_{i 3}\right)=(1,1,2)$, and $\left(c_{i 1}, c_{i 2}, c_{i 3}\right)=(2,1,1)$. Then if $s=1$, we have $M^{*}-\hat{M}=4-2>\Delta=p_{i 1}=1$.

Now consider any given iteration of the Benders algorithm. Let $J_{h i}$ be the set of jobs assigned to machine $i$ in a previous iteration $h$, and $M_{h i}^{*}$ the corresponding minimum tardiness incurred by machine $i$. The solution of the current master problem removes job $j \in J_{h i}$ from machine $i$ when $x_{i j}=0$. Thus by Lemma 1 , the resulting minimum makespan for machine $i$ is reduced by at most

$$
\sum_{j \in J_{i}^{h}} p_{i j}\left(1-x_{i j}\right)
$$


This provides lower bounding function $M_{h i}^{*}-\sum_{j \in J_{i}^{h}} p_{i j}\left(1-x_{i j}\right)$ for each machine $i$, which yields the Benders cuts (b) in the master problem:

$$
\begin{array}{ll}
\operatorname{minimize} & z \\
\text { subject to } & \sum_{i} x_{i j}=1, \text { all } j \\
& z \geq M_{h i}^{*}-\sum_{j \in J_{i}^{h}}\left(1-x_{i j}\right) p_{i j}, \text { all } i, h=1, \ldots, H-1(b) \\
& z \geq \frac{1}{C_{i}} \sum_{j} c_{i j} p_{i j} x_{i j}, \text { all } i
\end{array}
$$

where $x_{i j} \in\{0,1\}$. The relaxation (c) is similar to that for the minimum cost problem.

\section{Computational Results}

We solved randomly generated problems with MILP (using CPLEX), CP (using the ILOG Scheduler), and the logic-based Benders method. All three methods were implemented with OPL Studio, using the OPL script language. The CP problems, as well as the CP subproblems of the Benders method, were solved with the assignAlternatives and setTimes options, which result in substantially better performance.

Random instances were generated as follows. The capacity limit was set to $C_{i}=10$ for each facility $i$. For each task $j, c_{i j}$ was assigned the same random value for all facilities $i$ and drawn from a uniform distribution on $[1,10]$. For instances with $n$ tasks and $m$ facilities, the processing time $p_{i j}$ of each task $j$ on facility $i$ was drawn from a uniform distribution on $[i, 10 i]$. Thus facility 1 tends to run about $i$ times faster than facility $i$ for $i=1, \ldots, m$. Since the average of $10 i$ over $m$ facilities is $5(m+1)$, the total processing time of all tasks is roughly proportional to $5 n(m+1)$, or about $5 n(m+1) / m$ per facility. The release dates were set to zero, and the deadline for every task was set to $5 \alpha n(m+1) / m$ (rounded to the nearest integer). We used $\alpha=1 / 3$, which results in a deadline that is loose enough to permit feasible solutions but tight enough so that tasks are reasonably well distributed over the facilities in minimum cost solutions. In minimum cost problems, the cost $f_{i j}$ is drawn from a uniform distribution on $[2(m-i+1), 20(m-i+1)]$, so that faster facilities tend to be more expensive.

No precedence constraints were used, which tends to make the scheduling portion of the problem more difficult.

Table 1 displays computational results for 2,3 and 4 facilities as the number of tasks increases. The CP solver is consistently faster than MILP, and in fact MILP is not shown for the makespan problems due to its relatively poor performance. However, $\mathrm{CP}$ is unable to solve most problems with more than 16 tasks within two hours of computation time. 
Table 1. Computation times in seconds for minimum cost and minimum makespan problems, using MILP, CP, and logic-based Benders methods. Each time represents the average of 5 instances. Computation was cut off after two hours (7200 seconds), and $\mathrm{a}+$ indicates that this occurred for at least one of the five problems.

\begin{tabular}{|c|c|c|c|c|c|c|}
\hline \multirow{2}{*}{\multicolumn{2}{|c|}{ Facilities Tasks }} & \multicolumn{3}{|c|}{ Min cost } & \multicolumn{2}{|c|}{ Min makespan } \\
\hline & & MILP & $\mathrm{CP}$ & Benders & $\mathrm{CP}$ & Benders \\
\hline \multirow[t]{6}{*}{2} & 10 & 1.9 & 0.14 & 0.09 & 0.80 & 0.08 \\
\hline & 12 & 199 & 2.2 & 0.06 & 4.0 & 0.39 \\
\hline & 14 & 1441 & 79 & 0.04 & 299 & 7.8 \\
\hline & 16 & $3604+^{a}$ & 1511 & 1.1 & 3737 & 30 \\
\hline & 18 & & $7200+$ & 7.0 & $7200+$ & 461 \\
\hline & 20 & & & 85 & & \\
\hline \multirow[t]{8}{*}{3} & 10 & 0.86 & 0.13 & 0.37 & 0.85 & 0.06 \\
\hline & 12 & 797 & 2.6 & 0.55 & 7.5 & 0.3 \\
\hline & 14 & 114 & 35 & 0.34 & 981 & 0.7 \\
\hline & 16 & $678^{a}$ & 1929 & 4.5 & 4414 & 6.5 \\
\hline & 18 & & $7200+$ & 14.6 & $7200+$ & 13.3 \\
\hline & 20 & & & 2.9 & & 34 \\
\hline & 22 & & & 23 & & $1509^{b}$ \\
\hline & 24 & & & 53 & & \\
\hline \multirow[t]{8}{*}{4} & 10 & 2.0 & 0.10 & 0.6 & 0.07 & 0.09 \\
\hline & 12 & 7.2 & 1.4 & 4.0 & 1.9 & 0.09 \\
\hline & 14 & 158 & 72 & 2.8 & 524 & 0.8 \\
\hline & 16 & $906^{a}$ & 344 & 0.8 & 3898 & 0.9 \\
\hline & 18 & & $6343+$ & 5.2 & $7200+$ & 13.9 \\
\hline & 20 & & & 2.6 & & 25 \\
\hline & 22 & & & 22 & & 472 \\
\hline & 24 & & & 114 & & \\
\hline
\end{tabular}

${ }^{a}$ CPLEX ran out of memory on one or more problems, which are omitted from the average time.

${ }^{b}$ Average over three problems.

The Benders method is substantially faster than both CP and MILP. Its advantage increases rapidly with problem size, reaching some three orders of magnitude relative to $\mathrm{CP}$ for 16 tasks. Presumably the advantage would be greater for larger problems.

As the number of tasks increases into the 20 s, the Benders subroblems reach a size at which the computation time for the scheduling subproblem dominates and eventually explodes. This point is reached later when there are more facilities, since the subproblems are smaller when the tasks are spread over more facilities.

In practice, precedence constraints or other side constraints often accelerate the solution of the scheduling subproblems. Easier subproblems could allow the Benders method to deal with larger numbers of tasks. This hypothesis was tested by adding precedence constraints to the problem instances described above; see Table 2. This resulted in fast solution of the scheduling subproblems, except in the three largest makespan instances, which we omit because they do not test 
Table 2. Computational results for minimum cost and minimum makespan problems on two facilities with precedence constraints, using the Benders method. Computation time and number of iterations are shown for individual problem instances. Computation was cut off after 600 seconds. Minimum makespans are also given, except when computation is terminated prematurely, in which case lower and upper bounds are shown. In such cases a feasible solution with makespan equal to the upper bound is obtained.

\begin{tabular}{|c|cc|rrr|} 
Tasks & \multicolumn{2}{|c|}{ Minimum Cost } & \multicolumn{3}{|c|}{ Minimum Makespan } \\
& sec & iter. & makespan & sec & iter. \\
\hline 12 & 0.02 & 3 & 17 & 0.2 & 14 \\
14 & 0.05 & 5 & 13 & 0.3 & 17 \\
16 & 0.5 & 24 & 27 & 1.6 & 32 \\
18 & 0.02 & 15 & 27 & 25 & 152 \\
20 & 0.9 & 39 & 37 & 0.7 & 13 \\
22 & 0.7 & 34 & $26-27$ & 600 & 480 \\
24 & 7.7 & 115 & 32 & 13 & 92 \\
26 & 2.1 & 24 & 37 & 442 & 268 \\
28 & 21 & 162 & $35-37$ & 600 & 351 \\
30 & 73 & 261 & $50-53$ & 600 & 206 \\
32 & $>600$ & $>666$ & & & \\
34 & $>600$ & $>602$ & & & \\
36 & 235 & 318 & & & \\
\hline
\end{tabular}

the hypothesis. Easier subproblems in fact allow solution of somewhat larger instances. We also obtained good quality bounds on optimal makespan even when computation was terminated early. This approach that can be used for problems that are too large to solve to optimality.

Table 3 investigates how the Benders method scales up to a larger number of facilities, without precedence constraints. The average number of tasks per facility is fixed to 5 . The random instances are generated so that the fastest facility is roughly twice as fast as the slowest facility, with the other facility speeds spaced evenly in between.

Since the subproblems remain relatively small as the problem size increases, it is possible to accommodate more tasks than in Table 1.

\section{Conclusions and Future Research}

We find that logic-based Benders decomposition can substantially improve on the state of the art when solving minimum-cost and minimum-makespan planning and scheduling problems, in the latter case when all tasks have the same release date and deadline.

In the case of minimum makespan probems, the Benders approach has the additional advantage that it can be terminated early while still yielding both a feasible solution and a lower bound on the optimal makespan. The bound improves steadily as the algorithm runs.

Several issues remain for future research: 
Table 3. Computation times in seconds and number of iterations for minimum cost and minimum makespan problems, using the Benders method. Each figure represents the average of 5 instances.

\begin{tabular}{|c|c|c|c|c|c|}
\hline \multicolumn{2}{|c|}{ Tasks Facilities } & \multicolumn{2}{|c|}{$\begin{array}{c}\text { Min cost } \\
\text { sec } \quad \text { iterations }\end{array}$} & \multicolumn{2}{|c|}{$\begin{array}{l}\text { Min makespan } \\
\text { sec iterations }\end{array}$} \\
\hline 10 & 2 & 0.1 & 9 & 0.2 & 10 \\
\hline 15 & 3 & 0.7 & 15 & 1.6 & 25 \\
\hline 20 & 4 & $50^{a}$ & 57 & 13 & 37 \\
\hline 25 & 5 & 2.9 & 18 & 213 & 82 \\
\hline 30 & 6 & 4.8 & 26 & $2075^{c}$ & 103 \\
\hline 35 & 7 & 128 & 62 & & \\
\hline 40 & 8 & $976^{b}$ & 108 & & \\
\hline
\end{tabular}

${ }^{a}$ Includes one outlier that ran for $240 \mathrm{sec}$ and 191 iterations.

${ }^{b}$ One problem was terminated after $3600 \mathrm{sec}$ and 98 iterations.

${ }^{c}$ Average of 2 problems.

- Can effective Benders cuts be developed for minimum makespan problems in which tasks have different release dates and deadlines?

- Can a Benders method be applied for other objectives, such as minimum tardiness or minimum number of late tasks?

- Would a branch-and-check approach to the Benders method described here significantly improve performance on planning and scheduling problems?

- Would access to "dual" information from the CP scheduler (results of edge finding, etc.) result in more effective Benders cuts, and how would these cuts be derived?

\section{References}

1. Benders, J. F., Partitioning procedures for solving mixed-variables programming problems, Numerische Mathematik 4 (1962): 238-252.

2. Eremin, A., and M. Wallace, Hybrid Benders decomposition algorithms in constraint logic programming, in T. Walsh, ed., Principles and Practice of Constraint Programming (CP 2001), Lecture Notes in Computer Science 2239, Springer (2001).

3. Geoffrion, A. M., Generalized Benders decomposition, Journal of Optimization Theory and Applications 10 (1972): 237-260.

4. Hooker, J. N., Logic-based Methods for Optimization: Combining Optimization and Constraint Satisfaction, John Wiley \& Sons (2000).

5. Hooker, J. N. and G. Ottosson. Logic-based Benders decomposition, Mathematical Programming 96 (2003) 33-60.

6. Hooker, J. N., and Hong Yan, Logic circuit verification by Benders decomposition, in V. Saraswat and P. Van Hentenryck, eds., Principles and Practice of Constraint Programming: The Newport Papers, MIT Press (Cambridge, MA, 1995) 267-288.

7. Jain, V., and I. E. Grossmann, Algorithms for hybrid MILP/CP models for a class of optimization problems, INFORMS Journal on Computing 13 (2001) 258-276.

8. Thorsteinsson, E. S., Branch-and-Check: A hybrid framework integrating mixed integer programming and constraint logic programming, Lecture Notes in Computer Science 2239 (2001) 16-30. 
9. Türkay, M., and I. E. Grossmann, Logic-based MINLP algorithms for the optimal synthesis of process networks, Computers and Chemical Engineering 20 (1996) 959978. 